\title{
Performance analysis of ultra-reliable short message decode and forward relaying protocols
}

\author{
Parisa Nouri* (D), Hirley Alves and Matti Latva-aho
}

\begin{abstract}
Machine-type communication (MTC) is a rapidly growing technology which covers a broad range of automated applications and propels the world into a fully connected society. Two new use cases of MTC are massive MTC (mMTC) and ultra-reliable low latency communication (URLLC), where mMTC supports a large number of devices with high reliability and low rate connectivity while URLLC refers to excessively low outage probability under very stringent latency constraint. Herein, we examine the URLLC through three cooperative schemes, namely dual-hop decode and forward, selection combining, and maximum ratio combining, and compare to direct transmission under Rayleigh fading. We compare the performance of studied cooperative protocols under two distinct power constraints with respect to latency and energy efficiency. Moreover, we illustrate the impact of coding rate on the probability of successful transmission in ultra-reliable region in addition to the effect of power allocation on the outage probability. We also provide the performance analysis of cooperative schemes in terms of energy efficiency and latency requirements.
\end{abstract}

Keywords: Machine-type communications, Ultra-reliable low latency communication, Energy efficiency, Cooperative diversity, Outage probability, Rayleigh fading

\section{Introduction}

The fifth generation (5G) of cellular networks for beyond 2020 envisages to handle two new use cases in machinetype communications (MTC), namely ultra-reliable low latency communications (URLLC) and massive MTC (mMTC) [1]. In MTC, MTC devices autonomously communicate with minimum human cooperation $[2,3]$. 5G communication technology should be flexible enough to support ultra-reliable low latency communications by guaranteeing reliability greater than $99.999 \%$ [4]. Key challenges and requirements of $5 \mathrm{G}$ technology such as latency, data rate, energy, and cost issues are discussed in more details in $[1,5,6]$.

In recent years, MTC has gained much attention from the mobile network operators, equipment vendors, and academic researchers due to such novel communication paradigm, the capability of exchanging short data messages and also being cost-effective, energy efficient $[7,8]$,

*Correspondence: Parisa.Nouri@oulu.fi

Centre for Wireless Communications (CWC), University of Oulu, Oulu, Finland reliable, and within a stringent delay requirement. MTC takes advantage of several distinctive properties such as group-based communications, low mobility, and timecontrolled, time-tolerant, and secure connection which are at the same time challenging tasks since technically advanced solutions are needed to deliver the required tasks. Within the application requirements, hence, opening up different research areas is currently being carried out in academia, industry, and standard bodies [9]. Current technologies cover a small range of applications and services while the upcoming MTC should be able to cover a broad range of services with multiple forms of data traffic in order to deal with different service requirements as data rate, latency, reliability, energy consumption, and security $[6,10]$. Future MTC improvements will be conspicuous in health care, logistics, process automation, transportation, e.g., $[6,11]$. In mMTC, a huge number of devices in a specific domain are connected to the cellular network with low-rate and low-power connectivity, different quality-of-service (QOS) requirements, and high 
reliability to support demanding situations, e.g., smart meters and actuators $[12,13]$.

Moreover, MTC services have to met stringent timing constraints from few seconds to even excessively low endto-end deadlines in mission critical communications [14], connection between vehicles, remote control of robots in addition to an extreme low end-to-end latency in the scope of less than a millisecond which is a key enabler in several services including cloud connectivity, industrial control, and road safety $[1,11,15]$. Latency refers to the time duration between transferring the message from the transmitter and receiving correctly at the receiver where some messages drop due to the buffer overflows, unsuccessful synchronizations, and unsuccessful decoding which result in unlimited delay [1]. Hence, we can define the reliability as the probability of successful transmission under the predetermined delay constraint $[1,16]$. In URLLC, high probability of successful transmission indicates low outage probability (or packet drop) while the opposite does not always hold as the reliability is restricted to a specific latency budget due to the limited amount of channel uses [1]. Hence, one of the major requirements of URLLC is an extremely low outage probability under a very demanding latency budget where retransmissions are not always available. In the use of short messages under URLLC, new robust channel codes are needed; otherwise, the performance of the system will be even further away from the Shannon limit with long data packets [17].

Under Shannon's channel coding theorem, error-free communication is attained when the blocklength goes to infinity [18]. For instance, authors in [19] provide a tight approximation of achievable coding rate under finite blocklength $(\mathrm{FB})$ regime and indicate a noticeable performance loss compared to the Shannon coding. This motivates us to analyze the performance of MTC under FB regime since in URLLC, due to the equal packet length of metadata and information bits, an unsuccessful encoding of the metadata decreases the system efficiency [15]. In the past few years, several works have studied different aspects of FB coding since majority of the theoretical results assume infinite blocklength (IFB). For instance, authors in [20] examine some possible FB coding schemes which may be applied in 5G technology. They show that novel coding schemes with better minimum distance between the codewords improve the efficiency of system at the cost of more sophisticated decoders. Moreover, the performance of spectrum sharing networks with FB codes are studied in [21]. The blocklength of information bits highly affects the system quality where an optimal power allocation technique improves the system efficiency with short message transmissions. Furthermore, authors in [22] propose a new power allocation technique, so-called modified water-filling in order to maximize the lower bound of the coding rate with short packet transmission compared to the common water-filling method. In addition, performance of ARQ protocol in terms of throughput and average latency is studied in [23]. Authors determine the optimal lengths of the codeword which minimize the latency and maximize the throughput per-user for a specific number of information bits. They illustrate that with optimal codes, the shorter the codeword is, the lower outage probability attains.

\subsection{On the impact of cooperative diversity}

Cooperative diversity provides the possibility of high data rate while improving the reliability. In cooperative networks, intermediate nodes transfer the message from the source to the destination [24]. Cooperative technique exploits the spatial diversity gain to reduce the impact of wireless fading from multipath propagation. The major advantage of this technique is that the several independent copies of a signal arrive at the destination without installing collocated antennas at the source or receiver in addition of a better signal quality, better coverage, greater capacity, and lower transmit power $[25,26]$. The most conventional cooperative scheme is decode and forward (DF), where the auxiliary node, namely relay, decodes, encodes, and retransmits the message [25]. Cooperative schemes are categorized as fixed, adaptive, and feedback schemes [25]. In the fixed protocol, relay always forwards the message to destination while in adaptive protocol, the relay retransmits the message under a predefined threshold rule which enables that to communicate independently or not. In the feedback protocol, if the destination requests, the cooperation takes place [25]. During the past few years, the efficiency of cooperative networks has been investigated in several system and channel models. Authors in [27] propose a method that meet the high reliability and latency requirements through taking the advantage of cooperative relaying technique. Moreover, authors in [26] provide a comprehensive study regarding the exiting cooperative schemes and analyze the performance of each scheme. Relaying performance of quasistatic Rayleigh channels where the channel gains of the direct link and relaying are combined at the destination is studied in [18]. They indicate that the performance loss increases if the outage probability of the source-to-relay link is higher than the overall outage probability. The efficiency of multi-relay DF scenario under the assumption of perfect channel state information (CSI) and partial CSI is provided in [28]. Authors show that with perfect CSI, the throughput of IFB is smaller than the throughput of FB coding. Authors in [29] examine the throughput of a multi-hop relaying network under FB and IFB regimes with two assumptions: (i) target overall outage probability and (ii) constant coding rate. They illustrate that there is different but optimal number of hops which maximize 
the throughput for either FB or IFB assumptions. In addition, they indicate that the FB throughput is quasi-concave in the overall outage probability and coding rate. Furthermore, authors illustrate that the multi-hop network is less affected by the blocklength under the constant coding rate assumption compared to the target overall outage probability scenario. Moreover, [30] studies the performance of DF relay network in dissimilar Rayleigh fading channels. Although authors attain the closed form expression of the outage probability, they do not consider the impact of finite blocklength coding. Furthermore, authors in [31] study the achievable coding rate and ergodic capacity of non-orthogonal amply-and-forward (AF) multi-relay network subject a total average power constraint (TAPC) and an individual average power constraint (IAPC). They indicate that the ergodic capacity can be attained by an iterative water-filling-based algorithm. In addition, they show that in a multi-relay NAF network, the transmit power at the source should be equally allocated in all broadcasting phases to cover the capacity at sufficiently high SNRs.

Moreover, our previous work [32] introduces relaying as means to achieve ultra-reliability. We study the performance of cooperative relaying protocols, supposing Rayleigh fading channels. We show that relaying technique improves the reliability and how we can meet the ultra-reliable communication requirements. We examine the impact of coded blocklength and number of information bits on the probability of successful transmission. In addition, it is shown that relaying requires less transmit power compared to the direct transmission (DT) to enable ultra-reliability under FB regime. We also provide an approximation to the outage probability in closed form. We extend our work in [32], by considering ultrareliable MTC with incremental relaying technique in [33]. We define the overall outage probability in each studied relaying scheme, assuming Nakagami- $m$ fading. We investigate the impact of fading severity and power allocation factor on the outage probability. We also provide the outage probability in closed form. Our works in [32,33] show that cooperative diversity is useful to meet URLLC requirements.

\subsection{Energy efficiency of cooperative communication}

Another key characteristic of wireless communications that highly affect the performance of $5 G$ networks is the energy efficiency (EE) due to the limited energy resources in energy-constraint networks [34, 35]. EE, which has been widely studied recently in literatures, is defined as the ratio of successfully transmitted bits to the total consumed energy [36, 37]. Hence, reducing the amount of energy per bit improves EE at low SNR regime [35], particularly in wireless networks where the batteries which are not rechargeable or easy to charge supply the wireless components [36]. The reason which motivates us to study EE in the context of URLLC is that URLLC is achieved at the cost of high transmit power [38, 39], but we aim to show that cooperative diversity alleviates these demands.

In early works, authors in [37] examine EE in tactile Internet under queuing and transmission delays to design an energy-efficient resource allocation strategy. They propose an optimal resource allocation strategy where the average total consumed power under stringent latency constraint equals to that with unlimited queuing latency requirement with plenty of transmit antennas. Moreover, [36] provides a comprehensive overview of energyefficient networks and determines the trade-off between energy efficiency and spectrum efficiency and their applications in $5 \mathrm{G}$ networks.

\subsection{Our contribution}

In this work, we further study three cooperative protocols, namely DF, selection combining (SC), and maximum ratio combining (MRC). Furthermore, we indicate the superiority of MRC over SC and DF protocols in terms of coding rate and reliability. We also show the optimal value of power allocation at the source and relay in each of studied protocols. Moreover, we examine the minimum latency and energy efficiency in cooperative schemes under two different power allocation constraints.

The following are considered the contributions of this work.

- We provide the general expression of the outage probability for each relaying scheme studied in this work.

- We extend the work in [18], by proposing the closed form expression for the outage probability.

- We extend our previous works in $[32,33]$ by studying the minimum latency and energy efficiency under two distinct power constraints, so-called (i) equal power allocation (EPA) and (ii) optimal power allocation (OPA) strategies which are allocated numerically.

- We provide the asymptotic analysis of studied cooperative schemes.

The rest of this paper is organized as follows. Section 2 presents the system model. Section 3 discusses the cooperative diversity and examines the outage probability in three cooperative schemes considered in this work, and Section 4.1 presents some numerical results regarding the performance of studied cooperative schemes under URR. Section 4.2 investigates the energy efficiency of considered cooperative schemes and presents some numerical results. Finally, Section 5 concludes the paper. The important abbreviation and symbols are listed in Table 1. 
Table 1 Summary of the functions and symbols

\begin{tabular}{|c|c|}
\hline bpcu & Bit per channel use \\
\hline CSI & Channel state information \\
\hline DF & Decode and forward \\
\hline DT & Direct transmission \\
\hline $\mathrm{EE}$ & Energy efficiency \\
\hline EPA & Equal power allocation \\
\hline FB & Finite blocklength \\
\hline IFB & Infinite blocklength \\
\hline mMTC & Massive machine-type communication \\
\hline MRC & Maximum ratio combining \\
\hline MTC & Machine-type communication \\
\hline OPA & Optimal power allocation \\
\hline PDF & Probability density function \\
\hline QOS & Quality of service \\
\hline RV & Random variable \\
\hline SC & Selection combining \\
\hline $\mathrm{SH}$ & Single hop \\
\hline SNR & Signal to noise ration \\
\hline URLLC & Ultra-reliable low latency communication \\
\hline URR & Ultra reliable region \\
\hline$f_{W}()$. & Probability density function \\
\hline $\mathrm{E}()$. & Expectation \\
\hline$Q()$. & Q-function \\
\hline$C(\rho)$ & Shannon capacity \\
\hline$V(\rho)$ & Channel dispersion \\
\hline $\mathrm{Q}^{-1}()$. & Inverse of Q-function \\
\hline e & Exponential Euler's number \\
\hline$y$ & Received signal \\
\hline$h$ & Fading channel \\
\hline w & AWGN noise \\
\hline k & Information bits \\
\hline$x_{i}$ & Transmitted signal \\
\hline$n$ & Number of channel uses \\
\hline $\log _{2}$ & Logarithm to the base 2 \\
\hline$n_{S}$ & Number of channel uses for source \\
\hline$n_{R}$ & Number of channel uses for relay \\
\hline$d_{S D}$ & Distance of source-destination link \\
\hline$d_{S R}$ & Distance of source-relay link \\
\hline$d_{R D}$ & Distance of relay-destination link \\
\hline E & Energy efficiency \\
\hline$P$ & Total power \\
\hline$P_{R}$ & Power of relay \\
\hline$P_{S}$ & Power of source \\
\hline$P_{T X}$ & Power of transmitter \\
\hline
\end{tabular}

Table 1 Summary of the functions and symbols (Continued)

\begin{tabular}{ll}
\hline$P_{R X}$ & Power of receiver \\
$P_{P A}$ & Power of amplifier \\
$P_{\text {succ }}$ & Probability of successful transmission \\
$P_{\max }$ & Maximum total power \\
$N_{0}$ & Power of noise \\
$\Omega$ & Instantaneous SNR \\
$\gamma$ & Average SNR \\
$\rho$ & Average power constraint \\
$\eta$ & Power allocation factor \\
$\mathcal{R}$ & Maximum coding rate \\
$\phi$ & Drain efficiency \\
$\epsilon$ & Outage probability \\
\hline
\end{tabular}

\section{Preliminaries}

\subsection{System model}

Figure 1 illustrates a DF relaying scenario including a source $S$, destination $D$, and a decode-forward relay $R$. We normalize the distance of $S$ to $D$ as $d_{S D}=1 \mathrm{~m}$ and that $R$ can move in a straight line between $S$ and $D$, while the distance between $S$ and $R$ is denoted by $d_{S R}=$ $\beta d_{S D}$ and the distance of the relaying link is denoted by $d_{R D}=(1-\beta) d_{S D}$. The links denoted by the following random variables $X, Y$, and $Z$ represent the $S-R, R-D$, and $S-D$ links respectively, and each transmission uses $n_{i}$ channel uses where $i \in\{S, R\}$. This means that $n_{S}$ channel uses in the broadcasting phase and $n_{R}$ channel uses for the relaying phase. In this scenario, first, $S$ sends the message to the $D$ and $R$ in the broadcasting phase and if $R$ successfully decodes the message, forwards it to the $D$ in the relaying phase [18]. The received signals in the broadcasting phase are denoted as $y_{1}$ and $y_{2}$, and only if $R$ collaborates with $S$, the received signal at $D$ is $y_{3}$ as follows [18]

$$
y_{1}=h_{1} x+w_{1}, \quad y_{2}=h_{2} x+w_{2}, \quad \text { and } \quad y_{3}=h_{3} x+w_{3} \text {, }
$$

where $x$ is the transmitted signal with power $P$ and $w_{i}$ is the AWGN noise with power $N_{0}=1$ where $i \in\{X, Y, Z\}$. Quasi-static Rayleigh fading channels in the $S-D, S-R$, and $R-D$ links are denoted as $h_{1}, h_{2}$, and $h_{3}$, respectively. In this work, we consider two distinct power constraints, namely (i) EPA where equal powers are allocated to $S$ and $R$ and (ii) OPA where total power of $S$ and $R$ is equal to the maximum power. In a DF-based relaying protocol, the instantaneous SNR depends on the total power constraint $P=P_{S}+P_{R}=\eta P+(1-\eta) P$, which is given by $\Omega_{Z}=\eta P\left|h_{1}\right|^{2} / N_{0}, \Omega_{X}=\eta P\left|h_{2}\right|^{2} / N_{0}$, and $\Omega_{Y}=(1-\eta) P\left|h_{3}\right|^{2} / N_{0}$, where $0<\eta \leqslant 1$ is the power allocation factor considered to provide a fair comparison between DT and cooperative transmissions and $\eta=0.5$ 


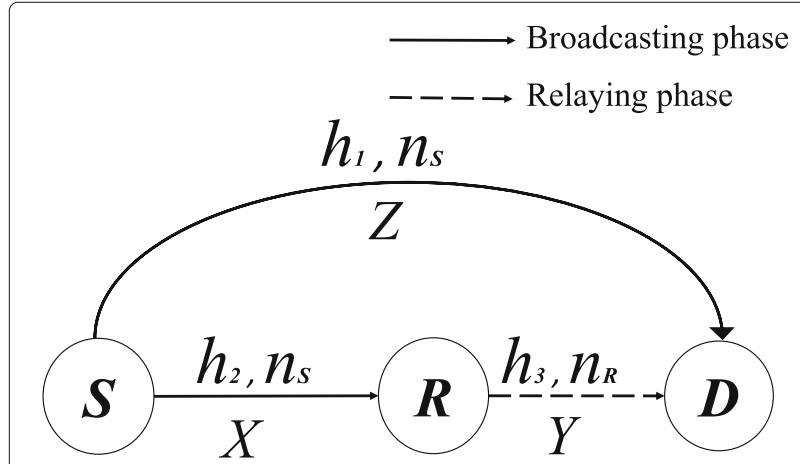

Fig. 1 System model for relaying with a source, destination, and a decode-forward relay. This figure illustrates the system model for cooperative decode-and-forward relaying scenario including a source, a relay, and a destination. The links between $S$ to $D, S$ to $R$, and $R$ to $D$ are referred as the direct link $h_{1}$, broadcasting link $h_{2}$ and relaying link $h_{3}$ each of which with $n_{i}$ channel uses respectively, where $i \in\{S, R\}$

with EPA. Hence, the average SNR in each link is $\gamma_{Z}=$ $\eta P / N_{0}, \gamma_{X}=\eta P / N_{0}$, and $\gamma_{Y}=(1-\eta) P / N_{0}$.

\subsection{Performance analysis of single-hop communication under the finite blocklength regime}

In this section, we revisit the concept of FB coding. In a single-hop communication, first, $k$ information bits are mapped to a sequence, namely codeword including $n$ symbols. Afterwards, the created codeword passes the wireless channels and channel outputs map into the estimate of the information bits. Thus, for a single-hop communication with blocklength $n$, outage probability $\epsilon$, and the average power constraint $\rho$, where $\frac{1}{n} \sum_{i}^{n}\left|x_{i}\right|^{2} \leq \rho$ holds, the maximum coding rate $\mathcal{R}^{*}(n, \epsilon)$ of AWGN channel in bits is calculated as

$$
\mathcal{R}^{*}(n, \epsilon)=C(\rho)-\sqrt{\frac{V(\rho)}{n}} \mathrm{Q}^{-1}(\epsilon) \log _{2} \mathrm{e},
$$

where $C(\rho)=\log _{2}(1+\rho)$ is the positive channel capacity and $V(\rho)=\rho(2+\rho) /(1+\rho)^{2}$ is the channel dispersion [15]. According to (2), the outage probability is given by

$$
\epsilon=\mathrm{Q}\left(\sqrt{n} \frac{C(\rho)-\mathcal{R}^{*}(n, \epsilon)}{\sqrt{V(\rho)} \log _{2} \mathrm{e}}\right)
$$

which holds for the AWGN channels where the channel coefficient $h_{i}$ is equal to one. While for quasi-static fading channels, we attain the outage probability as follows [15]

$$
\epsilon \approx \mathrm{E}\left[\mathrm{Q}\left(\sqrt{n} \frac{C\left(\rho|h|^{2}\right)-\mathcal{R}^{*}(n, \epsilon)}{\sqrt{V\left(\rho|h|^{2}\right)}}\right)\right] .
$$

Note that (4) is accurate for $n>100$, as proved for AWGN channels [19, Figures 12 and 13], as well as for fading channels as discussed in [40]. In addition, in the relaying schemes, we assume that $S$ can encode $k$ information bits into $n_{S}$ channel uses, while $R$ uses $n_{R}$ channel uses. Hence, $S$ and $R$ could employ more sophisticated encoding technique than $[18,41]$.

\subsection{Closed-form expression of the outage probability}

Unfortunately, (4) does not have a closed-form expression, but it can be tightly approximated as we shall see next.

\section{Lemma 1 The outage probability is approximated as}

$$
\epsilon=1-\frac{\zeta}{\sqrt{2 \pi}} \exp \left(-\theta_{m}\right)\left[\exp \left(\sqrt{\frac{\pi}{2 \zeta^{2}}}\right)-\exp \left(-\sqrt{\frac{\pi}{2 \zeta^{2}}}\right)\right]
$$

where $\theta_{m}=\frac{2^{\mathcal{R}}-1}{P}$ and $\zeta=P \sqrt{2 \pi} \mu$, where $\mu=$ $\sqrt{\frac{n}{2 \pi}}\left(e^{2 \mathcal{R}}-1\right)^{-\frac{1}{2}}$.

Proof Let us first define $g(x)=\sqrt{n} \frac{C(\rho)-\mathcal{R}}{\sqrt{V(\rho)}}$, then we resort to a linearization of the Q-function $[21,42]$

$$
K(t) \approx Q(g(x))= \begin{cases}1 & t \leqslant \varrho \\ \frac{1}{2}-\frac{\mu}{\sqrt{2 \pi}}(x-\theta) & \varrho<t<\vartheta \\ 0 & t \geq \vartheta\end{cases}
$$

where $\theta=2^{\mathcal{R}}-1, \vartheta=\theta+\sqrt{\frac{\pi}{2} \mu^{-2}}, \varrho=\theta-\sqrt{\frac{\pi}{2} \mu^{-2}}$. Then, we calculate $\mathrm{E}_{\mathrm{X}}[\mathrm{Q}(g(x))]=\int_{0}^{\infty} K(t) f_{X}(x) d x$, where $f_{X}(x)$ is the probability density function (PDF) of the SNR of the link $X$, and the solution is given in (5).

Remark Moreover, we compare the accuracy of linearized Q-function in (6) to original Q-function in (3) as indicated in Fig. 2. The difference between these two plots does not have a noticeable impact on the outage probability since we find the approximated outage probability in (5) via integrating over the SNR range and due to the symmetric property of the function as evinced by Fig. 2, regions that show the difference between the original and linearized Q-function, cancel each other and so, this difference becomes negligible as illustrated in Fig. 3. Thus, we can notice that error defined by error $=\left|\frac{\epsilon-\epsilon_{\text {app }}}{\epsilon}\right|$ is approximately equal to zero which shows the accuracy of the linearized Q-function applied in the closed form expression of the outage probability. Fore example, the maximum error over the entire SNR range is about $0.03 \%$. Similar conclusion holds for other values of $\mathcal{R}$.

\section{The proposed method}

In this section, we investigate the outage probability of cooperative $\mathrm{DF}, \mathrm{SC}$, and $\mathrm{MRC}$ protocols under the $\mathrm{FB}$ 


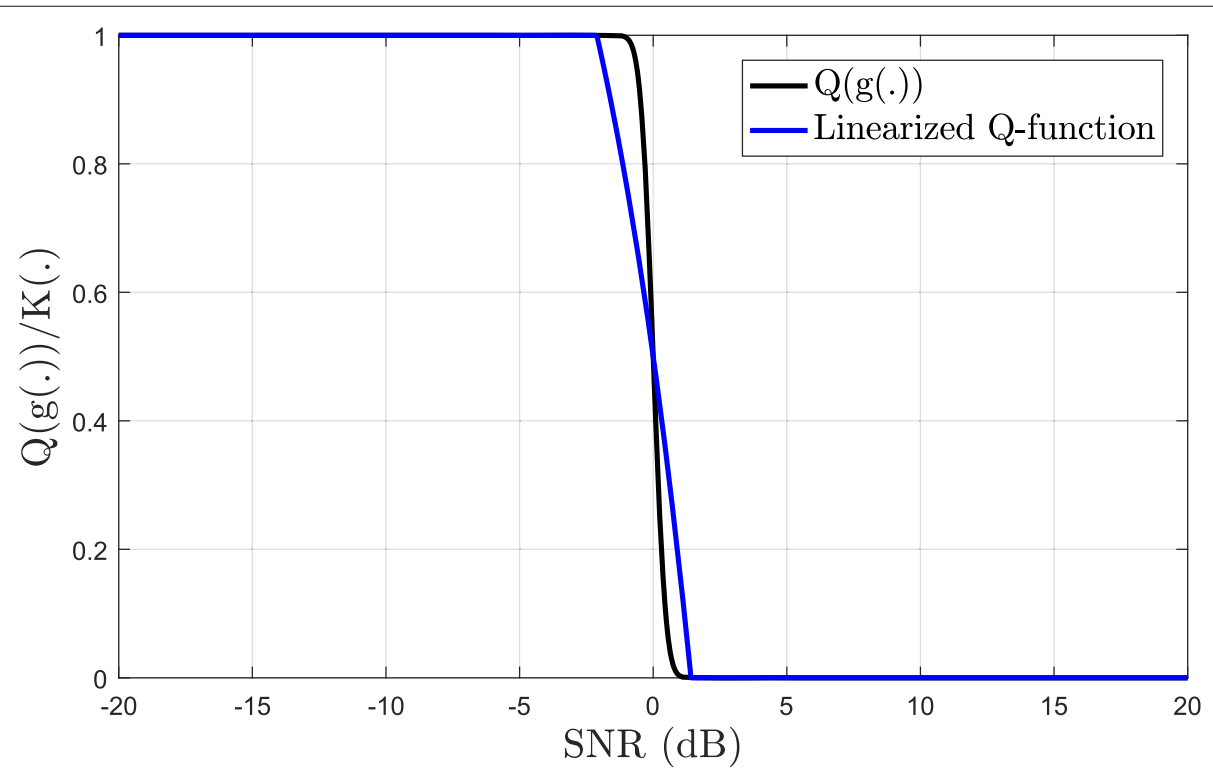

Fig. 2 Accuracy of linearized Q-function compared to the original Q-function. This figure illustrates the accuracy of Q-function linearization applied in closed form expression of outage probability

regime. The direct transmission model is used here as the basis of the comparison.

\subsection{Direct transmission}

The source sends the message directly to the destination, where $\Omega_{Z^{\prime}}=Z / \eta=P\left|h_{S D}\right|^{2} / N_{0}$, with average SNR $\gamma_{Z^{\prime}}=P / N_{0}$, where the outage probability is calculated as in (5) but $\zeta$ with $P_{S}=P$ and $\mu$ with $n=n_{S}$.

\subsection{Dual hop decode and forward (DF)}

In this scheme, since the $S-D$ distance is too large, it assumes that the direct link is in the outage; thus, $R$ always collaborates with the source. Hence, $S$ sends the message to both $R$ and $D$ in the broadcasting phase. Then, $R$ transfers the message to $D$ [43]. The overall outage probability is given by

$$
\epsilon_{D F}=\epsilon_{S R}+\left(1-\epsilon_{S R}\right) \epsilon_{R D}
$$

where $\epsilon_{S R}$ and $\epsilon_{R D}$ are calculated according to (5). Notice that we update $\zeta$ with $P_{S}=\eta P, P_{R}=(1-\eta) P$ and $\mu$ with $n=n_{S}, n=n_{R}$, respectively. This scenario can be analyzed as selection combining (SC) or maximum

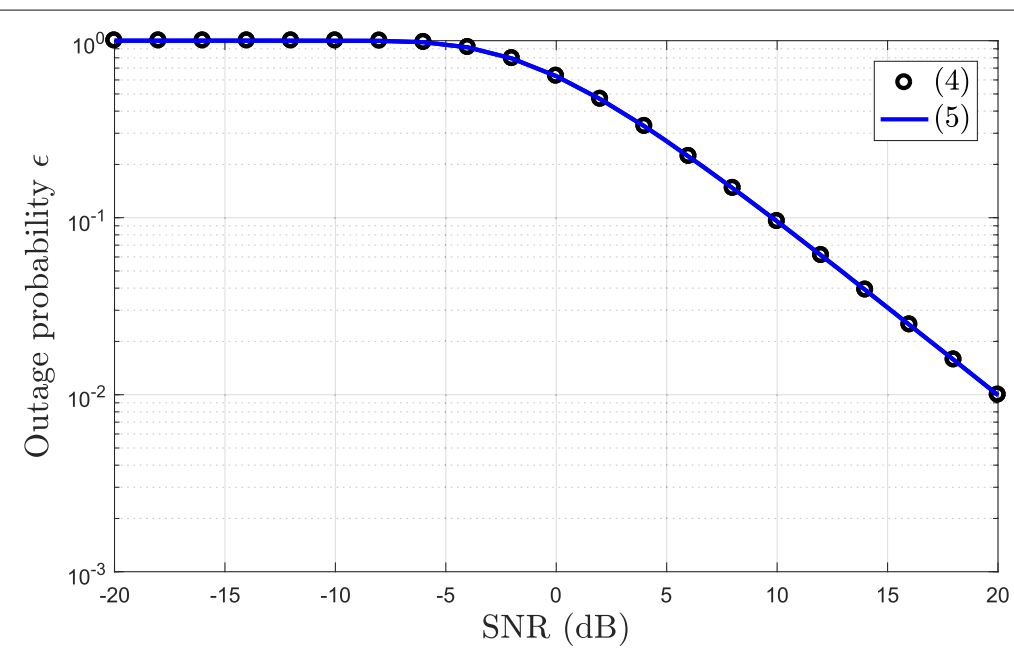

Fig. 3 Comparing the accuracy of approximated outage probability in (4) and (5). This figure compares the accuracy of outage probability with numerical integral of Q-function in (4) to the approximated outage probability in (5) 
ratio combining (MRC) depending on how the destination combines the original transmitted signal and the retransmitted signal.

\subsection{Selection combining (SC)}

In this protocol, $R$ starts to collaborate with $S$ if the destination confirms that the source transmission was unsuccessful and so, the destination requests for retransmission from $R$ to receive the frame correctly. Cooperation occurs if $R$ decodes the received message from $S$ correctly and so transfers the message to $D$. Thereafter, if $D$ confirms that the transmission from $R$ is also failed, $D$ requests for the next subsequent message from $S$. Thus, the outage probability happens only if both $S-D$ and $R-D$ links are in outage $[44,45]$. The overall outage is given by

$$
\epsilon_{S C}=\epsilon_{S D} \epsilon_{S R}+\left(1-\epsilon_{S R}\right) \epsilon_{S D} \epsilon_{R D}
$$

where $\epsilon_{S D}$ is equal to (5) where $\zeta$ is updated with $P_{S}=\eta P$ and $\mu$ with $n=n_{S}$.

\subsection{Maximum ratio combining (MRC)}

In this scenario, relay always collaborates with the source and so, the channel gains of $S-D$ and $R-D$ links are combined at the receiver. Thus, the aggregated SNR is bigger than the primary attempted transmission rate as the $S-D$ transmission failed. In addition, the outage probability occurs if $S-D$ or $R-D$ transmission fails. Hence, the instantaneous SNR is $\Omega_{W}=\Omega_{Z}+\Omega_{Y}[44,45]$. The outage probability is [44]

$$
\epsilon_{M R C}=\epsilon_{S D}\left(\epsilon_{S R}+\left(1-\epsilon_{S R}\right) \frac{\epsilon_{S R D}}{\epsilon_{S D}}\right)
$$

where $\epsilon_{S R D}$ is the outage probability of the source-torelay-to destination link, notice that the term $\frac{\epsilon_{S R D}}{\epsilon_{D F}}$ refers to the probability that $D$ was not able to decode $S$ message alone. In order to calculate the (9), first we need to attain the PDF of $W$, and then, we calculate the outage probability as proposed in Proposition 1. To do so, let $W$ denote the sum of two independently distributed exponential random variables (RV), $Z$ and $Y$. Then, $f_{W}(w)$ is [44]

$$
f_{W}(w)= \begin{cases}\frac{w}{\Omega_{Z}^{2}} \exp \left(-\frac{w}{\Omega_{Z}}\right) & \Omega_{Z}=\Omega_{Y} \\ \frac{\exp \left(-\frac{w}{\Omega_{Z}}\right)-\exp \left(-\frac{w}{\Omega_{Y}}\right)}{\Omega_{Z}-\Omega_{Y}} & \Omega_{Z} \neq \Omega_{Y}\end{cases}
$$

Since the RVs are independent, the proof is straightforward solution of $f_{W}(w)=\int_{0}^{\infty} f_{Z}(w-y) f_{y}(y) \mathrm{d} y$ [46].

Proposition 1 The outage probability of the MRC of the $S-D$ and $R-D$ links $\epsilon_{S R D}$, is equal to
$\epsilon_{S R D}=\left\{\begin{array}{l}\frac{2-\mathrm{e}^{-\varphi}+\mathrm{e}^{-\alpha}}{2}-\frac{\rho \mathrm{e}^{-\varphi}}{\Omega_{Z}}+\frac{\rho \mathrm{e}^{-\varphi}-\vartheta \mathrm{e}^{-\alpha}}{2 \Omega_{Z}}+\frac{\mu \theta \Delta+2 \mu \xi}{\sqrt{2 \pi}}+\frac{(\mu \tau) / \Omega_{Z}}{\sqrt{2 \pi}} \Omega_{Z}=\Omega_{Y} \\ \frac{\Omega_{Z}-\Omega_{Y}+\Omega_{Z} \mathrm{e}^{-\alpha} \lambda_{1}+\Omega_{Z} \mathrm{e}^{-\varphi_{\lambda}+\Omega_{Y} \mathrm{e}^{-\frac{\vartheta}{\Omega_{Y}}}} \lambda_{3}+\Omega_{Y} \mathrm{e}^{-\frac{\rho}{\Omega_{Y}}} \lambda_{4}}{\Omega_{Z} \Omega^{-\Omega_{Y}}} \Omega_{Z} \neq \Omega_{Y}\end{array}\right.$

$$
\begin{gathered}
\tau=\vartheta^{2} \mathrm{e}^{-\frac{\vartheta}{\Omega_{Z}}}-\varrho^{2} \mathrm{e}^{-\frac{\varrho}{\Omega_{Z}}}-\theta \vartheta \mathrm{e}^{-\frac{\vartheta}{\Omega_{Z}}}+\theta \varrho \mathrm{e}^{-\frac{\varrho}{\Omega_{Z}}}, \\
\xi=\vartheta \mathrm{e}^{-\frac{\vartheta}{\Omega_{Z}}}-\varrho \mathrm{e}^{-\frac{\varrho}{\Omega_{Z}}}+\Omega_{Z} \mathrm{e}^{-\frac{\vartheta}{\Omega_{Z}}}-\Omega_{Z} \mathrm{e}^{-\frac{\varrho}{\Omega_{Z}}},
\end{gathered}
$$

where, $\vartheta$ and $\varrho$ are specified in (6), and $\lambda_{1}=$ $\frac{\mu \vartheta+\mu \Omega_{Z}-\mu \theta}{\sqrt{2 \pi}}-\frac{1}{2}, \lambda_{2}=\frac{\mu \theta-\mu \rho-\mu \Omega_{Z}}{\sqrt{2 \pi}}-\frac{1}{2}, \lambda_{3}=\frac{1}{2}-$ $\frac{\mu \vartheta+\mu \Omega_{Y}-\mu \theta}{\sqrt{2 \pi}}, \lambda_{4}=\frac{1}{2}+\frac{\mu \varrho+\mu \Omega_{Y}-\mu \theta}{\sqrt{2 \pi}}, \Delta=\mathrm{e}^{-\varphi}-\mathrm{e}^{-\alpha}$, $\varphi=\frac{\varrho}{\Omega_{Z}}$ and $\alpha=\frac{\vartheta}{\Omega_{Z}}$.

Proof By plugging (10) into (5) and multiplying by the linearized Q-function $K(t)$, we attain

$$
\epsilon=\int_{0}^{\varrho} f_{X}(x) d x+\int_{\varrho}^{\vartheta}\left(\frac{1}{2}-\frac{\mu}{\sqrt{(2 \pi)}}(x-\theta)\right) f_{X}(x) d x,
$$

which is solved with help of ([47], Eq. 2.321) and after some algebraic manipulations we attain (11) [32].

\subsection{Asymptotic analysis}

The outage probability in (5) can be defined as $\mathrm{P}\left[\Omega_{i} \leq \gamma_{\text {th }}\right]$ as the SNR goes to infinity, where $\gamma_{\text {th }}=2^{\overline{\mathcal{R}}}-1$. Thus, the approximated asymptotic outage probability per link in Rayleigh fading channels is $\epsilon_{i}=1-\exp \left(-\gamma_{\mathrm{th}} / \gamma_{i}\right)$, where $\gamma_{i}$ is a function of $\beta$ and $P$ ([38], \$10). Thereafter, we resort to Taylor series as $\gamma_{\text {th }} / \gamma_{i}$ approaches zero as SNR $\rightarrow \infty$, and so, $\exp (x) \approx 1+x$ and attain an asymptotic expression as $\epsilon_{i} \approx\left(\gamma_{\text {th }} / \gamma_{i}\right)$ ([38], $\$ 11)$. The asymptotic expression of $\epsilon$ after maximum ratio combining of $S$ and $R$ transmissions $\epsilon_{\mathrm{SRD}}$ is given in ([44], \$7). Therefore, the outage probability is approximated as $\epsilon_{\mathrm{SRD}}^{\infty} \approx \frac{\gamma_{\mathrm{th}}^{2}}{2 \gamma_{Z} \gamma_{Y}}$, resorting to series expansion as $\gamma_{i} \rightarrow \infty$.

\section{Numerical results and discussion}

\subsection{URLLC via cooperative diversity}

In this section, we show some numerical results of cooperative relaying transmission under FB regime. First, we show the impact of coding rate on the probability of successful transmission where MRC protocol outperforms DF, SC, and DT in terms of reliability. We also indicate the minimum latency required to support URLLC. Thereafter, we show the optimal value of power allocation factor $\eta$ for each of studied protocols. In addition, we compare the performance of cooperative relaying to DT in terms of power consumption and blocklength to perform under the UR region (URR). We verify the accuracy 
of our analytical model through the Monte-Carlo simulations. Unless stated otherwise, assume maximum transmit power per link as $20 \mathrm{~dB}, n=500, k=500$, and $R$ is in between $S$ and $D$, with $\beta=\frac{1}{2}$. The URR is shaded purple area in the following plots, and its most loose constraint is denoted with a red line where the outage probability is $10^{-3}$; thus, $1-\epsilon=99.9 \%$ reliability is feasible.

\subsubsection{Reliability vs. coding rate}

Figure 4 compares the probability of successful transmission $\left(P_{\text {succ }}=1-\epsilon\right)$ as a function of coding rate in URR. We can clearly see that MRC supports URLLC with higher coding rates compared to DT, DF, and SC schemes which is more evident with short packet lengths under the FB regime. Hence, MRC is less affected by the coding rate growth under the URR. For instance, with $n=400$ and $\mathcal{R}=0.43$, MRC covers $99.999 \%$ reliability, while SC provides equal reliability as MRC but with lower coding rate as $k=153$ and $n=400$. In addition, with $n=400$, reliability decreases to $99.9 \%$ and $99 \%$ with $k=133$ and $k=293$ for DF and DT schemes, respectively. Thus, URLLC is feasible via the cooperative schemes and we can apply each of these schemes based on our requirements such as reliability, packet length, and number of transmitted information bits.

In Fig. 5, we examine the impact of power allocation factor $\eta$ on the probability of successful transmission. As mentioned earlier in Section 2, in order to provide a fair comparison between DT and cooperative schemes, we allocate powers to $S$ and $R$ according to the power allocation factor. In DF, outage probability is minimized via equal power allocation strategy while in SC and MRC, we exploit additional diversity of the direct link; thus, less power should be allocated to $R$ as shown in Fig. 5. We also illustrate that URLLC is feasible through the cooperative schemes, particularly with SC and MRC where the outage probability is minimized to 0.2 and $0.1 \%$, respectively. As we indicated in our previous work in [32], these results holds for other values of SNR and coding rate.

In Fig. 6, we compare the ultra-reliable performance of cooperative schemes to DT in terms of transmit power under equal power allocation constraint. We can clearly see the power gain attained via the cooperative protocols at high SNR regime where there is huge performance gap between cooperative schemes and DT. In addition, we indicate that MRC and SC protocols perform closely in the entire SNR range and consumes less transmit power to communicate under the URR in comparison to DF and DT.

In addition, we indicate the possibility of using asymptotic expressions in ultra-reliable region. In other words, at high SNR regime, the maximum achievable coding rate (2) converges the asymptotically long codewords as $\mathcal{R}_{\text {asym }}(n, \epsilon)=C_{\epsilon}$, where $C_{\epsilon}=\sup \left\{\mathcal{R}: \operatorname{Pr}\left[\log _{2}(1+\rho)<\right.\right.$ $\mathcal{R}]<\epsilon$. In Fig. 6, we show that the asymptotic expressions approach the analytical results as the transmit power increases.

Figure 7 indicates the performance advantage of cooperative schemes over DT. Cooperative schemes exploit diversity gain which decreases the outage probability remarkably. As we expected, the outage probability decreases in blocklength. In addition, SC and MRC protocols are able to support URLLC under FB regime with very short packet lengths.

Figure 8 indicates the total minimum latency required for URLLC under the FB regime with two distinct power constraints as (i) EPA: $P=\eta P+(1-\eta) P$, where $\eta=0.5$,
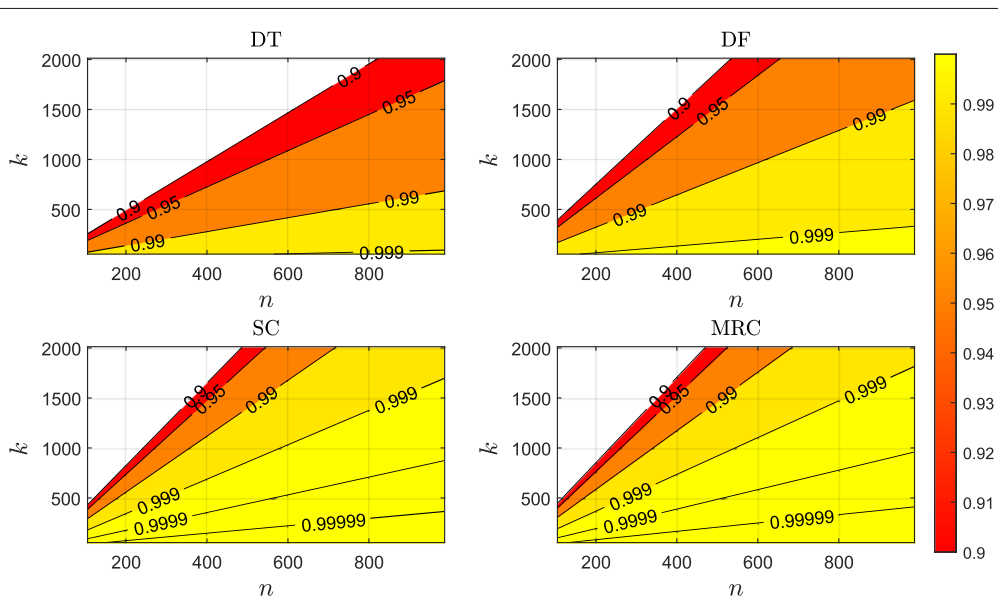

Fig. 4 Probability of successful transmission as a function $k$ and $n$. This figure compares the impact of coding of on the performance of cooperative schemes, namely DF, SC, and MRC to direct transmission. We show that MRC outperforms other studied cooperative scenarios in terms of reliability and coding rate 


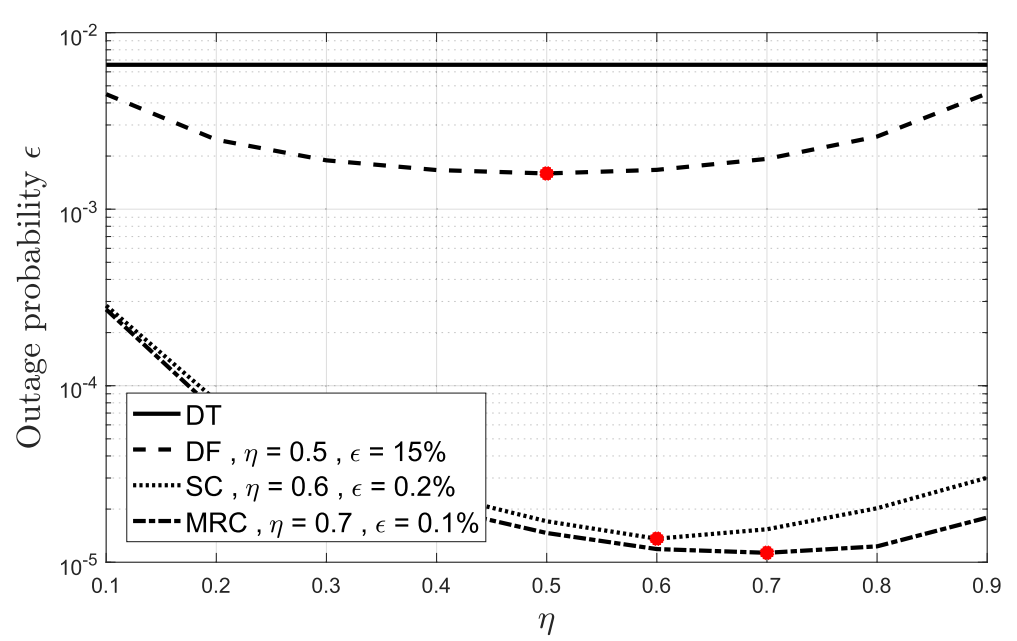

Fig. 5 Impact of power allocation factor $\eta$ on the outage probability with $k=250$. This figure illustrates the optimal value of power allocation factor for each of studied relaying scenarios where the outage probability is minimized. We show that we have to allocate more power to the source to work in ultra-reliable region and MRC requires more power at the source compared to the other studied protocols

and (ii) OPA: $P_{S}+P_{R} \leq P$. The choices of the minimum latency $\delta$ and optimal powers are in such a way that minimizes the outage probability constraint to a specific interval of interest and holds the power constraints which gives the optimal values of $n$ and $P$, and is a nonlinear optimization problem as follows. ${ }^{1}$

$$
\begin{array}{ll}
\text { minimize } & \epsilon(n, P) \\
\text { subject to } & P_{S}+P_{R} \leq P \\
& 100 \leq n \leq 10000
\end{array}
$$

We set the minimum blocklength to 100 since (4) is accurate for $n>100$, as proved for AWGN channels
([19], Figures 12 and 13) as well as for fading channels as discussed in [40], and to a maximum of $10^{4}$ so to reduce the search range, and to be within URLLC boundaries.

It shows that DT is not able to cope with the stringent latency constraint and need a large tolerance of delay; thus, we resort to cooperative protocols in order to reduce the latency in URR. It can be clearly seen that SC works highly better than DF and performs closely to MRC in the entire range but with higher latency requirements when we allocate equal powers to the $S$ and $R$. We also indicate that under OPA constraint, SC outperforms MRC in terms of channel uses and is more energy efficient than $\mathrm{MRC}$ as we discuss about it in the following section, while

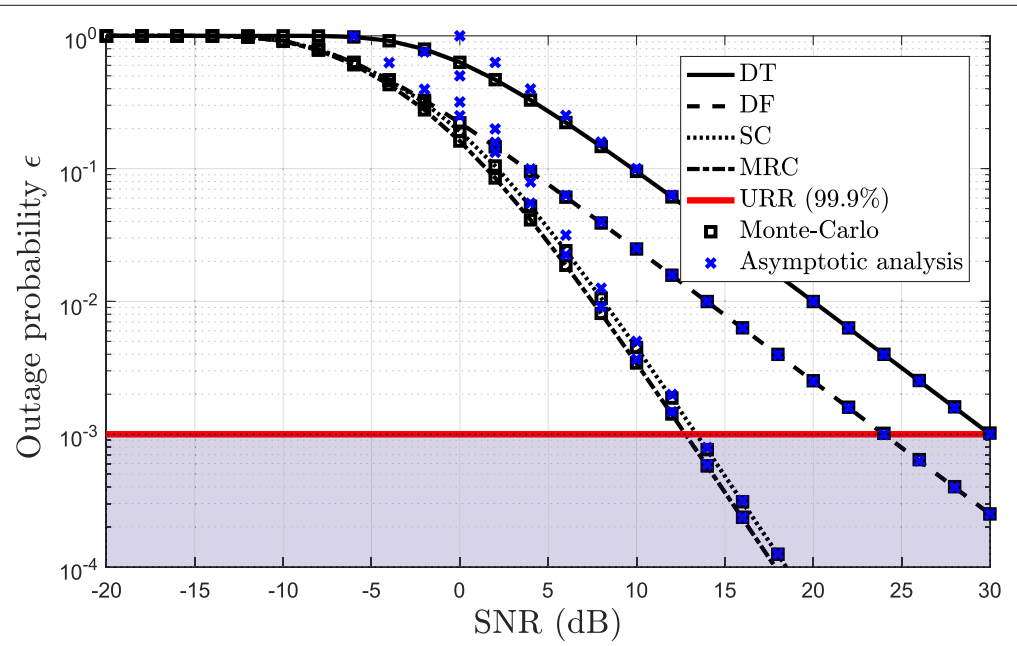

Fig. 6 Performance analysis of cooperative protocols under EPA. This figure compares the power consumption of studied cooperative schemes to direct transmission under the equal power allocation strategy. We show that under EPA, MRC requires less transmit power to work under ultra-reliable region compared to the other studied cooperative relaying protocols. Monte-Carlo simulations confirm the accuracy and appropriateness of our analytical model. In addition, asymptotic expressions approach the analytical results as the SNR increases 


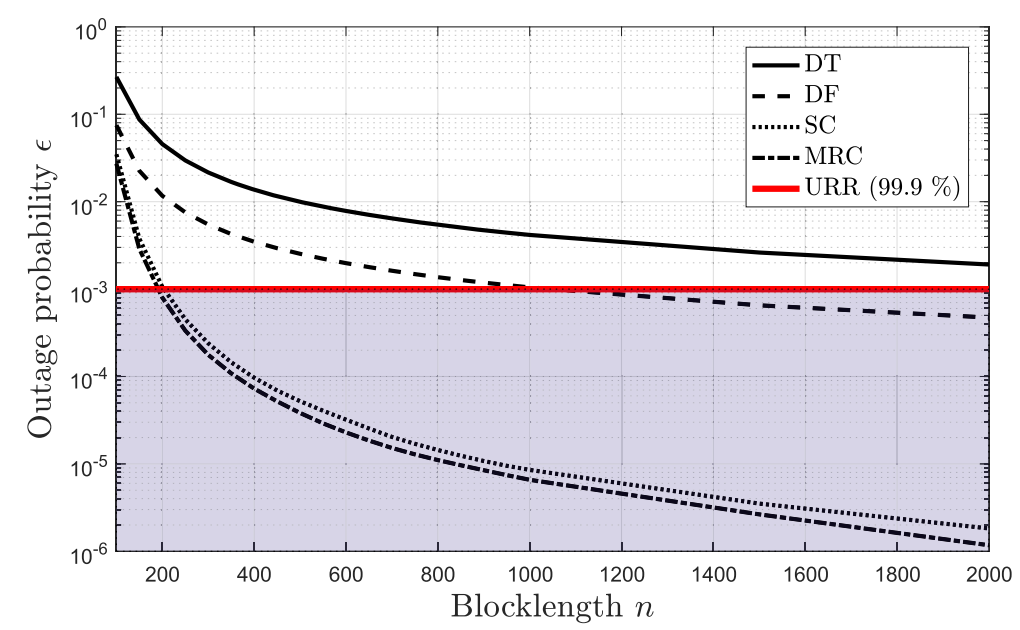

Fig. 7 Outage probability analysis for DT, DF, SC, and MRC under EPA. This figure compares the performance of cooperative schemes to direct transmission in terms of outage probability under the finite blocklength regime. We indicate that URLLC is feasible via cooperative diversity technique. We show the superiority of MRC and SC over DF and DT schemes under the finite blocklength regime

with equal power allocation strategy, MRC requires less channel uses and consumes less transmit power as we can see in Fig. 6. Here, with equal power allocation strategy, the total power of $S$ and $R$ may be less than the maximum total power $(20 \mathrm{~dB})$ but in Fig. 6, we force $P_{S}$ and $P_{R}$ to be equal with total power of $P_{\max }$. Therefore, according to the simulations, when $\mathcal{R}=1$ bpcu and $P=20 \mathrm{~dB}$, higher reliability is feasible in Fig. 6 compared to Fig. 8.

\subsection{Energy efficiency analysis}

Energy efficiency (EE) determines the trade-off between throughput gains and total energy consumed. Let us first define the total energy consumption per bit of each scenario. The total power consumed includes power of transmission with no dependency on the distance of relay nodes, consumed power in radio frequency(RF) circuitry and also coding rate $[48,49]$. Here, we ignore the baseband processing consumption since its value is negligible in comparison to the energy consumption of $\mathrm{RF}$ circuitry [50].

Then, the total energy consumption per bit of a singlehop transmission is

$$
\mathrm{E}_{\mathrm{SH}}=\frac{P_{P A}+P_{T X}+P_{R X}}{\mathcal{R}},
$$

where $P_{P A}=P / \phi$ is the power amplifier consumption for a single-hop transmission and $\phi$ is the drain efficiency of the amplifier, $P_{T X}$ and $P_{R X}$ are the power consumed

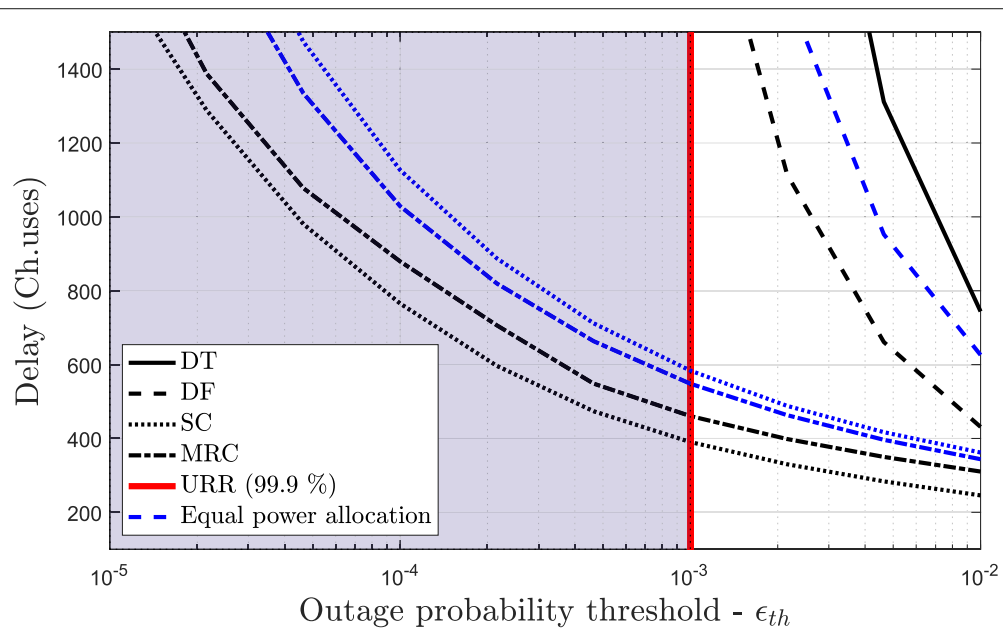

Fig. 8 Total latency in terms of channel uses with EPA and OPA constraints. This figure compares the total minimum latency in cooperative schemes under two distinct power constraints, so-called EPA and OPA. We show that under EPA, MRC has the lowest latency while with OPA, SC outperforms DF and MRC protocols in terms of latency requirement 
for transmitting and receiving in the internal circuitry, respectively. In a similar way, we can also find the total power consumption of multi-hop schemes by determining the outage probability on $S-R$ link in each cooperative schemes.

\subsubsection{Cooperative transmissions}

The total power consumption for DF protocol depends on the outage probability of $S-R$ link as follows

$\mathrm{E}_{\mathrm{DF}}=\epsilon_{S R_{\mathrm{DF}}} \times \frac{P_{P A}+P_{T X}+P_{R X}}{\mathcal{R}}+\left(1-\epsilon_{S R_{\mathrm{DF}}}\right) \times \frac{2 P_{P A}+2 P_{T X}+2 P_{R X}}{\mathcal{R}}$,

where the first term indicates that the consumed energy on the $S$ - $R$ link, while the second term shows that $R$ could decode the message correctly and send the packet to $D$.

In the case of SC and MRC, the total power consumption is formulated as follows

$$
\begin{aligned}
\mathrm{E}_{j}= & \epsilon_{S R_{j}} \times \frac{P_{P A}+P_{T X}+2 P_{R X}}{\mathcal{R}}+\left(1-\epsilon_{S R_{j}}\right) \\
& \times \frac{2 P_{P A}+2 P_{T X}+3 P_{R X}}{\mathcal{R}},
\end{aligned}
$$

where $j \in\{S C, M R C\}$ and $\epsilon_{S R}$ is calculated by (5) accordingly to each method. The additional $P_{R X}$ in each term of (17) compared to the (16) corresponds to the transmission of $S$, which is heard by both $R$ and $D$ and destination decodes $S-D$ and $R-D$ transmissions, simultaneously.

Hence, the EE for each protocol is formulated as

$E E=\frac{\text { Throughput }}{\text { Total energy consumed }}=\frac{\mathcal{R}(1-\epsilon)}{\mathrm{E}}, \quad$ (bits/Ch.uses/W)

Furthermore, as observed from Fig. 9, $E E(\epsilon, \mathrm{E})$ is nonconvex in the SNR, while the outage probability is monotonically decreasing in the SNR and energy consumption is monotonically increasing, which is observed in Figs. 6

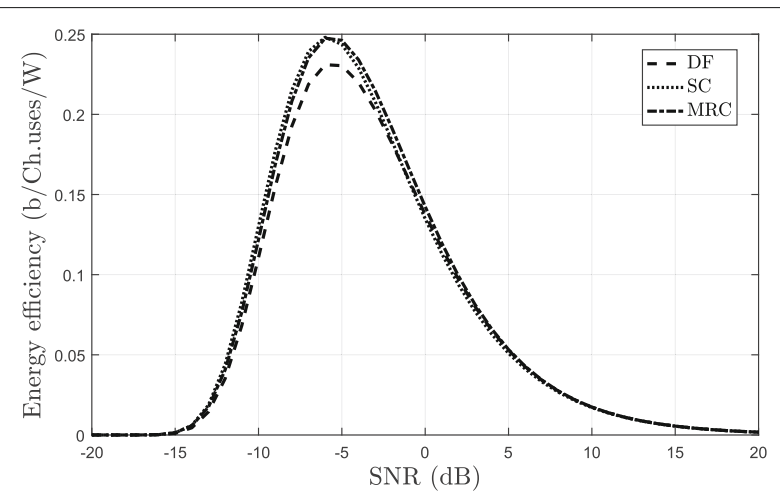

Fig. 9 Energy efficiency analysis of cooperative schemes under equal power allocation. This figure compares the energy efficiency in DF, SC and MRC schemes under EPA as a function maximum transmit power and 11, respectively. We maximize the energy efficiency as follows

$$
\begin{array}{ll}
\text { Maximize } & E E(\epsilon, E) \\
\text { subject to } & P_{S}+P_{R} \leq P, \\
& \epsilon(n, P) \leq \epsilon_{\mathrm{th}}, \\
& 100 \leq n \leq 10000 .
\end{array}
$$

This problem is equivalent to minimize the outage probability with respect to $P_{S}^{*}, P_{R}^{*}$, and blocklength $n^{*}$. Since we aim to compare the performance of cooperative schemes, we do not focus on the proposal of a particular solution, but we resort to numerically efficient algorithm. Therefore, we resort to $f_{\text {mincon }}$ implemented in Matlab and use interior point algorithm to solve the nonlinear optimization problem as detailed in [51]. We consider outage probability threshold in an interval of interest as $10^{-5}<\epsilon_{\text {th }}<10^{-2}$. At each outage probability value, we numerically determine $P_{S}^{*}, P_{R}^{*}$, and blocklength $n^{*}$ that maximize the energy efficiency. We apply the numerical optimization due to the nonlinear constraint on the outage probability $\epsilon(n, P) \leq \epsilon_{\text {th }}$.

Figure 10 compares the energy efficiency of cooperative schemes in terms of probability of successful transmission under two distinct power constraints. In this paper, we assume $P_{T X}=97.9 \mathrm{~mW}, P_{R X}=112.2 \mathrm{~mW}$, and the drain efficiency $\phi=0.35$ according to the power consumption values reported in [50]. Under EPA strategy, MRC is the most energy-efficient scenario among other cooperative scenarios as it consumes less transmit power shown in Fig. 6, and has lower latency in URR while under OPA, SC becomes the most energy-efficient protocol as we show in Fig. 8, it reduces the latency and the total power consumption is less than that of MRC. Since Fig. 5 indicates that in order to perform in URR, we should allocate more power to the source where $\eta$ is equal to 0.6 and 0.7 for $\mathrm{SC}$ and MRC, respectively. Hence, more power is allocated to the source of MRC than that of SC; thus, MRC becomes less energy efficient compared to SC under OPA strategy.

Figure 11 compares the total consumed energy in each of studied cooperative scenarios under EPA and OPA strategies. Under EPA, as we expected, MRC is superior and consumes less transmit power compared to DF and SC protocols, while with OPA, SC outperforms MRC and becomes most energy-efficient protocol. In addition, with the maximum transmit power of $20 \mathrm{~dB}$, no feasible solutions are found for DF protocol under stringent reliability requirements, which evinces the need for more sophisticated cooperative protocols. Feasible solutions are found if the transmit power increases, but it would be spectrally and energy insufficient. 


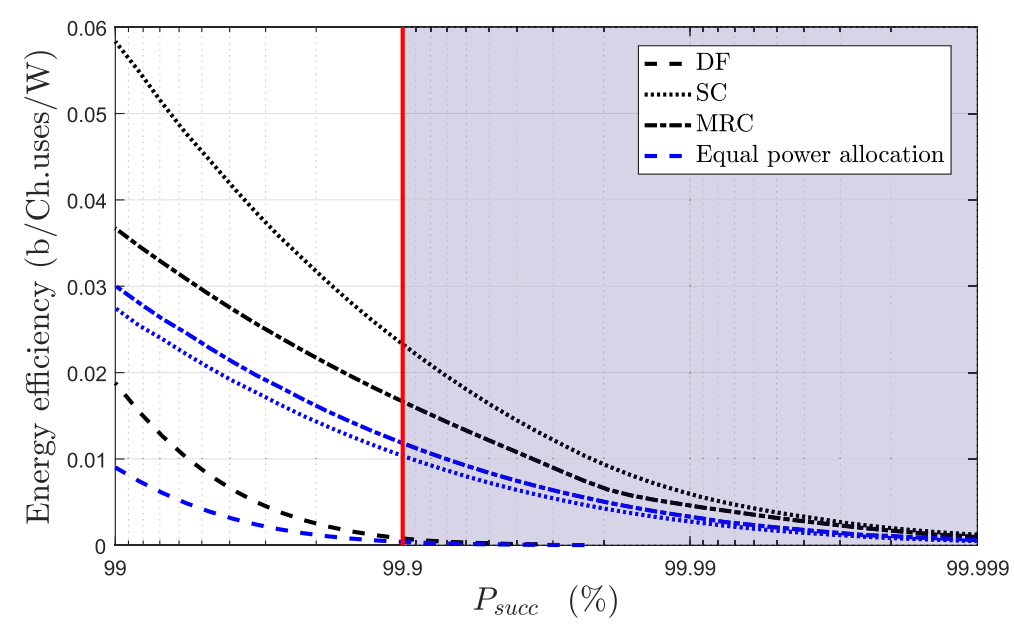

Fig. 10 Energy efficiency of DF, SC, and MRC scenarios under EPA and OPA strategies. This figure compares the energy efficiency in DF, SC and MRC cooperative schemes under EPA and OPA strategies as function of reliability. We show that under EPA, MRC is the most energy-efficient strategy while with OPA strategy, SC becomes the most energy-efficient protocol

\section{Conclusions}

In this paper, we assess the relay communication under the finite blocklength regime under Rayleigh fading. Performance of three relaying scenario, namely DF, SC, and $\mathrm{MRC}$ is compared to direct transmission under two distinct power constraints so-called EPA and OPA. Based on the outage probability analysis of each transmission protocol, we show that relaying improves the probability of a successful transmission and guarantees ultra-high reliability with FB codes. MRC protocol is less affected by the coding and provide higher reliability compared to DT and two other relaying scenarios. In addition, we numerically show the optimal power allocation for the relaying protocols under study in order to operate in URR. Our results shows that operation at URR is feasible by allocating more power to the source; however, relay node is considered to provide additional diversity gain compared to the DT which is more evident at high SNR regime. We compare the studied cooperative schemes in terms of latency and energy efficiency under the two distinct power constraints. According to the results, with equal power allocation at source and relay, MRC is the most energy-efficient protocol with lower latency and power consumption compared to the other scenarios while SC has the highest energy efficiency and lowest latency under optimal power allocation strategy. Moreover, we provide

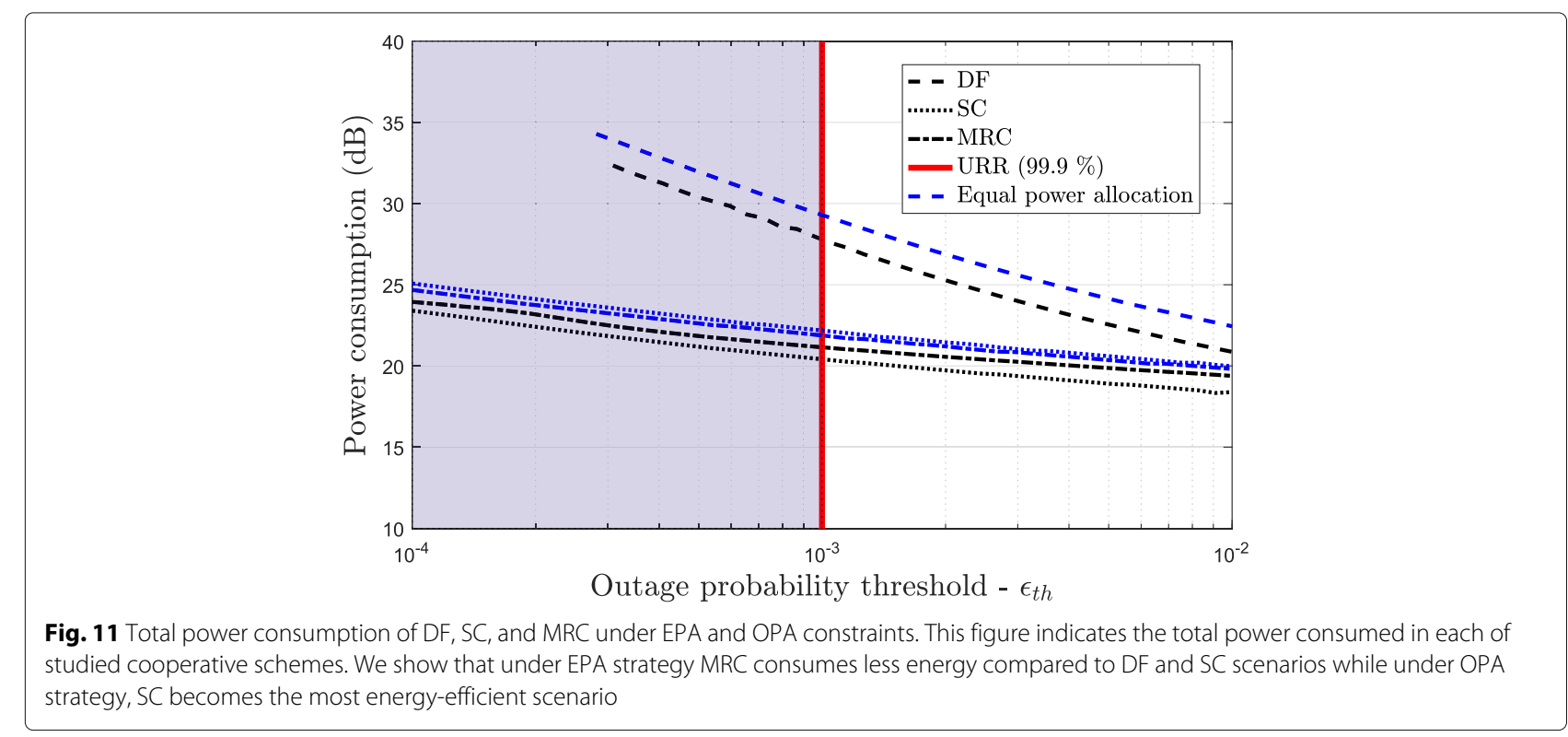


the outage probability in closed form and prove the accuracy and appropriateness of our analytical model through numerical results. Finally, in our future work, we will focus on the impact of imperfect channel state information on URLLC.

\section{Endnote}

${ }^{1}$ We solve the optimization problem numerically with the Matlab function fmincon. Interior point algorithm is used to solve the nonlinear optimization problem [51].

\section{Funding}

This work has been partially supported by Finnish Funding Agency for Technology and Innovation (Tekes), Huawei Technologies, Nokia and Anite Telecoms, and Academy of Finland (under Grant no. 307492)

\section{Availability of data and materials}

The manuscript is self-contained. Simulations description and parameters are provided in details in Section 4.

\section{Authors' contributions}

All authors have contributed to this manuscript and approved the final submitted manuscript.

\section{Competing interests}

The authors declare that they have no competing interests.

\section{Publisher's Note}

Springer Nature remains neutral with regard to jurisdictional claims in published maps and institutional affiliations.

Received: 16 October 2017 Accepted: 30 July 2018

Published online: 15 August 2018

\section{References}

1. P Popovski, JJ Nielsen, C Stefanovic, E de Carvalho, E Ström, KF Trillingsgaard, A-S Bana, DM Kim, R Kotaba, J Park, et al., Ultra-reliable low-latency communication (URLLC): principles and building blocks (2017). arXiv preprint arXiv:1708.07862

2. H Shariatmadari, R Ratasuk, S Iraji, A Laya, T Taleb, R Jäntti, A Ghosh, Machine-type communications: current status and future perspectives toward 5G systems. IEEE Commun. Mag. 53(9), 10-17 (2015)

3. Y Mehmood, C Görg, M Muehleisen, A Timm-Giel, Mobile M2M communication architectures, upcoming challenges, applications, and future directions. EURASIP J. Wirel. Commun. Netw. 2015(1), 250 (2015)

4. AE Kalør, R Guillaume, JJ Nielsen, A Mueller, P Popovski, Network slicing for ultra-reliable low latency communication in industry 4.0 scenarios (2017). arXiv preprint arXiv:1708.09132

5. JG Andrews, S Buzzi, W Choi, SV Hanly, A Lozano, AC Soong, JC Zhang, What will $5 \mathrm{G}$ be? IEEE J. Sel. Areas. Commun. 32(6), 1065-1082 (2014)

6. Z Dawy, W Saad, A Ghosh, JG Andrews, E Yaacoub, Toward massive machine type cellular communications. IEEE Wirel.Commun. 24(1), 120-128 (2017)

7. B Lee, S Park, DJ Love, H Ji, B Shim, in Global Communications Conference (GLOBECOM), 2016 IEEE. Packet structure and receiver design for low-latency communications with ultra-small packets (IEEE, Washington DC, 2016), pp. 1-6

8. TTaleb, A Kunz, Machine type communications in 3GPP networks: potential, challenges, and solutions. IEEE Commun. Mag. 50(3), 178-184 (2012)

9. M Condoluci, M Dohler, G Araniti, A Molinaro, K Zheng, Toward $5 \mathrm{G}$ densenets: architectural advances for effective machine-type communications over femtocells. IEEE Commun. Mag. 53(1), 134-141 (2015)

10. JF Monserrat, G Mange, V Braun, H Tullberg, G Zimmermann, Ö Bulakci, METIS research advances towards the $5 \mathrm{G}$ mobile and wireless system definition. EURASIP J. Wirel. Commun. Netw. 2015(1), 53 (2015)
11. C Bockelmann, N Pratas, H Nikopour, K Au, T Svensson, C Stefanovic, P Popovski, A Dekorsy, Massive machine-type communications in 5G: physical and MAC-layer solutions. IEEE Commun. Mag. 54(9), 59-65 (2016)

12. Popovski $P$, in $5 G$ for Ubiquitous Connectivity (5GU), 2014 1st International Conference on. Ultra-reliable communication in $5 \mathrm{G}$ wireless systems (IEEE, Akaslompolo, 2014), pp. 146-151

13. B Singh, O Tirkkonen, Z Li, MA Uusitalo, R Wichman, in Personal, Indoor, and Mobile Radio Communications (PIMRC), 2016 IEEE 27th Annual International Symposium on. Selective multi-hop relaying for ultra-reliable communication in a factory environment (IEEE, Valencia, 2016), pp. 1-6

14. A Biral, H Huang, A Zanella, M Zorzi, in Globecom Workshops (GC Wkshps), 2016 IEEE. On the impact of transmitter channel knowledge in energy-efficient machine-type communication (IEEE, Washington DC, 2016), pp. 1-7

15. G Durisi, T Koch, P Popovski, Toward massive, ultrareliable, and low-latency wireless communication with short packets. Proc. IEEE. 104(9), 1711-1726 (2016)

16. O Yilmaz, in European Conference on Networks and Communications (EUCNC), vol. 2016. Ultra-reliable and low-latency 5G communication (IEEE, Athens, 2016)

17. M Sybis, K Wesolowski, K Jayasinghe, V Venkatasubramanian, $\checkmark$ Vukadinovic, in Vehicular Technology Conference (VTC-Fall), 2016 IEEE 84 th. Channel coding for ultra-reliable low-latency communication in $5 \mathrm{G}$ systems (IEEE, Montreal, 2016), pp. 1-5

18. Y Hu, J Gross, A Schmeink, On the performance advantage of relaying under the finite blocklength regime. IEEE Commun. Lett. 19(5), 779-782 (2015)

19. Y Polyanskiy, HV Poor, Verdú S, Channel coding rate in the finite blocklength regime. IEEE Trans. Inf. Theory. 56(5), 2307-2359 (2010)

20. O Iscan, D Lentner, Xu W, in Globecom Workshops (GC Wkshps), 2016 IEEE. A comparison of channel coding schemes for $5 \mathrm{G}$ short message transmission (IEEE, Washington DC, 2016), pp. 1-6

21. B Makki, T Svensson, Zorzi M, in Communications (ICC), 2015 IEEE International Conference on. Finite block-length analysis of spectrum sharing networks (IEEE, London, 2015), pp. 7665-7670

22. J-H Park, D-J Park, A new power allocation method for parallel AWGN channels in the finite block length regime. IEEE Commun. Lett. 16(9), 1392-1395 (2012)

23. R Devassy, G Durisi, P Popovski, EG Strom, in Communications, Control and Signal Processing (ISCCSP), 2014 6th International Symposium on. Finite-blocklength analysis of the ARQ-protocol throughput over the Gaussian collision channel (IEEE, Athens, 2014), pp. 173-177

24. S Ikki, MH Ahmed, Performance analysis of cooperative diversity wireless networks over Nakagami-m fading channel. IEEE Commun. Lett. 11(4), 334-336 (2007)

25. E Zimmermann, P Herhold, G Fettweis, On the performance of cooperative relaying protocols in wireless networks. Trans. Emerg. Telecommun. Technol. 16(1), 5-16 (2005)

26. F Mansourkiaie, MH Ahmed, Cooperative routing in wireless networks: a comprehensive survey. IEEE Commun. Surv. Tutor. 17(2), 604-626 (2015)

27. VN Swamy, S Suri, P Rigge, M Weiner, G Ranade, A Sahai, B Nikolić, in Communications (ICC), 2015 IEEE International Conference on. Cooperative communication for high-reliability low-latency wireless control (IEEE, London, 2015), pp. 4380-4386

28. Y Hu, A Schmeink, Gross J, in Sensor Array and Multichannel Signal Processing Workshop (SAM), 2016 IEEE. Relaying with finite blocklength: challenge vs. opportunity (IEEE, Rio de Janerio, 2016), pp. 1-5

29. F Du, Y Hu, L Qiu, A Schmeink, in International Symposium on Wireless Communication Systems (ISWCS), 2016. Finite blocklength performance of multi-hop relaying networks (IEEE, Poznan, 2016), pp. 466-470

30. NC Beaulieu, J Hu, A closed-form expression for the outage probability of decode-and-forward relaying in dissimilar Rayleigh fading channels. IEEE Commun. Lett. 10(12), 813-815 (2006)

31. TX Tran, NH Tran, HR Bahrami, S Sastry, On achievable rate and ergodic capacity of NAF multi-relay networks with CSI. IEEE Trans. Commun. 62(5), 1490-1502 (2014)

32. P Nouri, H Alves, M Latva-aho, in European Conference on Networks and Communications (EUCNC), 2017. On the performance of ultra-reliable decode and forward relaying under the finite blocklength (IEEE, Oulu, 2017), pp. 1-5

33. P Nouri, H Alves, R Demo Souza, M Latva-aho, in International Symposium on Wireless Communication Systems (ISWCS), 2017. Ultra-reliable short 
message cooperative relaying protocols under Nakagami-m fading (IEEE, Bologna, 2017)

34. D Qiao, MC Gursoy, S Velipasalar, in Global Telecommunications Conference, 2009. GLOBECOM 2009. IEEE. Energy efficiency of fixed-rate wireless transmissions under queueing constraints and channel uncertainty (IEEE, Honolulu, 2009), pp. 1-6

35. MC Gursoy, On the capacity and energy efficiency of training-based transmissions over fading channels. IEEE Trans. Inf. Theory. 55(10), 4543-4567 (2009)

36. G Wu, C Yang, S Li, Li GY, Recent advances in energy-efficient networks and their application in 5G systems. IEEE Wirel. Commun. 22(2), 145-151 (2015)

37. C She, C Yang, in Communications in China (ICCC), 2016 IEEE/CIC International Conference on. Energy efficient design for tactile internet (IEEE, Chengdu, 2016), pp. 1-6

38. E Dosti, M Shehab, H Alves, M Latva-aho, in Networks and Communications (EuCNC), 2017 European Conference on. Ultra reliable communication via CC-HARQ in finite block-length (IEEE, Oulu, 2017), pp. 1-5

39. E Dosti, UL Wijewardhana, H Alves, M Latva-aho, Ultra reliable communication via optimum power allocation for type-I ARQ in finite block-length (2017). arXiv preprint arXiv:1701.08617

40. W Yang, G Durisi, T Koch, Y Polyanskiy, Quasi-static multiple-antenna fading channels at finite blocklength. IEEE Trans. Inf. Theory. $\mathbf{6 0}(7)$ 4232-4265 (2014)

41. Y Hu, A Schmeink, J Gross, Blocklength-limited performance of relaying under quasi-static Rayleigh channels. IEEE Trans. Wirel. Commun. 15(7), 4548-4558 (2016)

42. B Makki, T Svensson, M Zorzi, Finite block-length analysis of the incremental redundancy HARQ. IEEE Wirel. Commun. Lett. 3(5), 529-532 (2014)

43. JN Laneman, DN Tse, GW Wornell, Cooperative diversity in wireless networks: efficient protocols and outage behavior. IEEE Trans. Inf. Theory. 50(12), 3062-3080 (2004)

44. H Alves, RD Souza, G Fraidenraich, ME Pellenz, Throughput performance of parallel and repetition coding in incremental decode-and-forward relaying. Wirel. Netw. 18(8), 881-892 (2012)

45. H Alves, RD Souza, G Brante, ME Pellenz, in Vehicular Technology Conference (VTC Spring), 2011 IEEE 73rd. Performance of type-i and type-ii hybrid ARQ in decode and forward relaying (IEEE, Yokohama, 2011), pp. 1-5

46. A Papoulis, SU Pillai, Probability, random variables, and stochastic processes. (Tata McGraw-Hill Education, 2002)

47. IS Gradshteyn, IM Ryzhik, Table of Integrals, Series, and Products. (Academic press, United States, 2014)

48. GG de Oliveira Brante, MT Kakitani, RD Souza, Energy efficiency analysis of some cooperative and non-cooperative transmission schemes in wireless sensor networks. IEEE Trans. Commun. 59(10), 2671-2677 (2011)

49. H Alves, RD Souza, G Fraidenraich, Outage, throughput and energy efficiency analysis of some half and full duplex cooperative relaying schemes. Trans. Emerg. Telecommun. Technol. 25(11), 1114-1125 (2014)

50. S Cui, AJ Goldsmith, A Bahai, Energy-constrained modulation optimization. IEEE Trans. Wirel. Commun. 4(5), 2349-2360 (2005)

51. RA Waltz, JL Morales, J Nocedal, D Orban, An interior algorithm for nonlinear optimization that combines line search and trust region steps. Math. Program. 107(3), 391-408 (2006)

\section{Submit your manuscript to a SpringerOpen ${ }^{\circ}$ journal and benefit from:}

- Convenient online submission

- Rigorous peer review

- Open access: articles freely available online

- High visibility within the field

- Retaining the copyright to your article

Submit your next manuscript at $\gg$ springeropen.com 